\title{
Swept Source-Optical Coherence Tomography Angiography for Management of Secondary Choroidal Neovascularization in Punctate Inner Choroidopathy
}

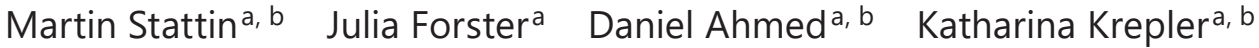 \\ Siamak Ansari-Shahrezaei ${ }^{a}, b, c$ \\ ${ }^{a}$ Karl Landsteiner Institute for Retinal Research and Imaging, Vienna, Austria; ${ }^{b}$ Department \\ of Ophthalmology, Rudolf Foundation Hospital, Vienna, Austria; 'Department of \\ Ophthalmology, Medical University of Graz, Graz, Austria
}

\begin{abstract}
Keywords
Intravitreal anti-vascular endothelial growth factor - Punctate inner choroidopathy · Secondary choroidal neovascularization - Swept source-optical coherence tomography angiography
\end{abstract}

\begin{abstract}
The purpose was to demonstrate the diagnostic and therapeutic feasibility of swept sourceoptical coherence tomography angiography (SS-OCTA) by picturing neovascular changes secondary to a rare white dot syndrome following long-term intravitreal ranibizumab (IVR). A 28-year-old Caucasian myopic female presented with visual loss in her right eye only. The clinical examination and multimodal imaging including spectral domain (SD)-OCT, blue-peak autofluorescence, fluorescein, and indocyanine green angiography (HRA Spectralis, Heidelberg Engineering; Heidelberg, Germany) as well as SS-OCTA (DRI Triton, Topcon; Tokyo, Japan) led to the diagnosis of idiopathic punctate inner choroidopathy with secondary subfoveal choroidal neovascularization (CNV). In addition to oral corticosteroids, a pro re nata regimen with IVR was initiated and guided by repeated SD-OCT and SS-OCTA. Six IVR were administered based on functional SS-OCTA en face scans illustrating vessel transformation and downsizing of the CNV area while SD-OCT B-scans were inconclusive as indirect signs of activity were absent throughout the follow-up period. SS-OCTA provided new possibilities for monitoring vessel development. IVR was managed based on vessel density as displayed by SS-OCTA.
\end{abstract}




\section{Introduction}

Punctate inner choroidopathy (PIC) is a multifocal chorioretinopathy of currently unknown pathogenesis [1]. It is regarded as a disease that predominantly affects young myopic women [2]. Characteristic funduscopic findings are sparsely small, yellow-grayish dots in the posterior pole with minimal or no vitreous inflammation [3]. Different stages of PIC lesions can be distinguished by optical coherence tomography (OCT), indicating the outer layers as the primary target of the disease [4]. The visual outcome strongly depends on the localization of the lesions besides other complications like secondary choroidal neovascularization (CNV) or subretinal fibrosis. The diagnosis of PIC is sometimes challenging and often delayed if secondary complications like CNV occurred. Therapeutic management in these cases varies and can be frustrating as no general recommendation exists to date [5]. Herein, a case of secondary CNV due to PIC was diagnosed with the help of swept source-OCT angiography (SS-OCTA) and successfully treated in a bilateral way under its surveillance.

\section{Case Presentation}

A 28-year-old Caucasian myopic female presented with visual loss and metamorphopsia in her right eye for 2 weeks in 2017. The patient gave her written informed consent to publish the case. Best-corrected visual acuity was 20/200 Snellen (Sn) on the right eye and 20/20 Sn on the left eye. The funduscopic appearance revealed several central bright dots next to submacular hemorrhage with minimal vitreous inflammation only in her right eye (Fig. 1a). Spectralis HRA-OCT Confocal Scanning Laser Ophthalmoscope and Angiography (Heidelberg Engineering, Heidelberg, Germany) was used for the acquisition of blue-peak fundus autofluorescence, spectral domain (SD)-OCT, fluorescein (FA), and indocyanine green angiography. Fundus autofluorescence set the hemorrhage apart from subretinal fluid (SRF) (Fig. 1b). FA showed early leakage consistent with CNV type 2 (Fig. 1c). Early indocyanine green angiography identified a subfoveal choroidal neovascular network and several
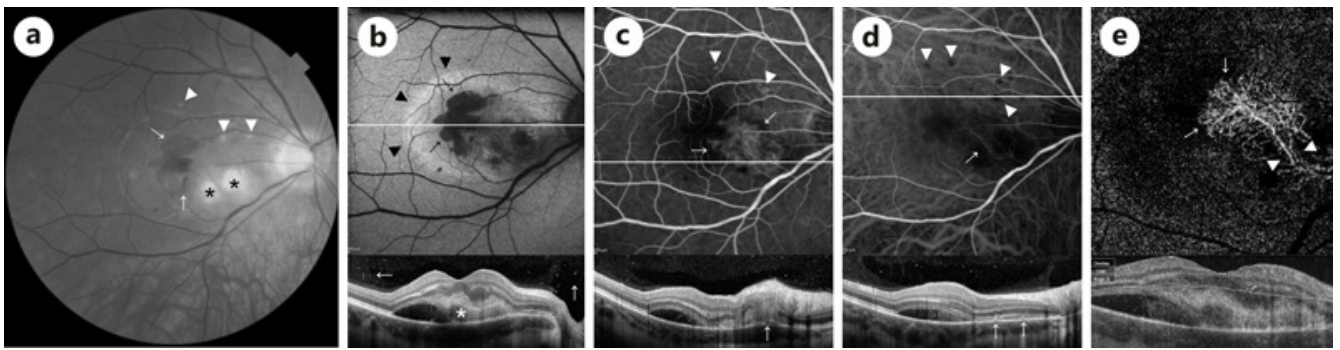

Fig. 1. a Multimodal imaging at presentation: infrared photography with hemorrhage $(\rightarrow)$ next to minor dots $(\Delta)$ and bright spots $(*)$. b Blue-peak FAF separating blood $(\rightarrow)$ from SRF $(\Delta)$ and a SD-OCT with vitreous cells $(\rightarrow)$; SRF mixed with subretinal hyperreflective material $\left(^{*}\right)$. c Early FA angiography of a subretinal CNV $(\rightarrow)$ besides ill-defined hyperfluorescence $(\Delta)$ and a SD-OCT with a RPE defect $(\rightarrow)$ due to a neovascular breakthrough. d Early indocyanine green angiography with the CNV $(\rightarrow)$ besides hypocyanescent dots $(\Delta)$ corresponding to defects of the RPE $(\rightarrow)$ in the SD-OCT. e Dense CNV with capillaries $(\rightarrow)$ and a feeder trunk $(\Delta)$ in a SS-OCTA image and its corresponding outer retina segmentation. FAF, fundus autofluorescence; SRF, subretinal fluid; SD-OCT, spectral domain-optical coherence tomography; FA, fluorescein; CNV, choroidal neovascularization; RPE, retinal pigment epithelium; SS-OCTA, swept source-optical coherence tomography angiography. 
well-circumscribed dots of hypocyanesence suggesting a deeper choroidal origin of the lesions (Fig. 1d). SD-OCT cross section B-scans illustrated a few scattered vitreous cells only at the posterior pole, neurosensory detachment caused by SRF and hyperreflective material next to a disintegration of the retinal pigment epithelium/photoreceptor junction. The Triton DRI SS-OCTA (Topcon Corporation, Tokyo, Japan) was utilized for OCTA en face and structural OCT B-scans. OCTA is able to distinguish between static and dynamic tissue by phase variance analysis. The advantage of the swept source technology relies on a higher wavelength $(1,050 \mathrm{~nm})$ with enhanced depth penetration and a faster scanning rate of currently $100,000 \mathrm{~A}$-scans/s [6]. The en face SS-OCTA $4.5 \times 4.5 \mathrm{~mm}$ isolated a dense vascular network with numerous capillary branches and anastomoses (Fig. 1e). Secondary CNV in PIC was diagnosed with the help of multimodal imaging. SS-OCTA isolated the CNV pattern expanding to the fovea. Treatment with intravitreal ranibizumab (IVR; $0.5 \mathrm{mg}$ in $0.05 \mathrm{~mL}$ ) in addition to oral corticosteroids (1 mg/kg/days) was initiated [5]. Subsequent SS-OCTA as well as SD-OCT images were conducted monthly for IVR administration in a pro re nata regimen. A complete resolution of blood was observed biomicroscopically and of SRF in the structural SD-OCT 1 month later (Fig. 2a, b), while downsizing of the CNV area and a reduction of vessel density could be displayed in SS-OCTA en face scans (Fig. 2c). Another IVR was performed due to persistent peripheral loops at the vessel termini, while the central feeder trunk was least affected [7]. Oral steroids were gradually tapered over 3 months without signs of PIC relapse. No signs of activity such as SRF on SD-OCT or funduscopic evidence of hemorrhage could be detected during the long-term period of nearly 3 years. In spite of that, SS-OCTA depicted differences in the neovascular complexity on repeated scans [8]. A total of 6 IVR were administered based on the growth or regression of new vessels at the border of the CNV lesion (Fig. 3a-h). At the last follow-up, best-corrected visual acuity was 20/20 Sn while SS-OCTA en face scans showed hyperdense vessel formation pointing toward the foveal umbo, suggesting either non- or very slow lesion progression (Fig. 3i-l). Continuous SS-OCTA scanning with traceable macular cubes will provide detailed information on future vascular changes.
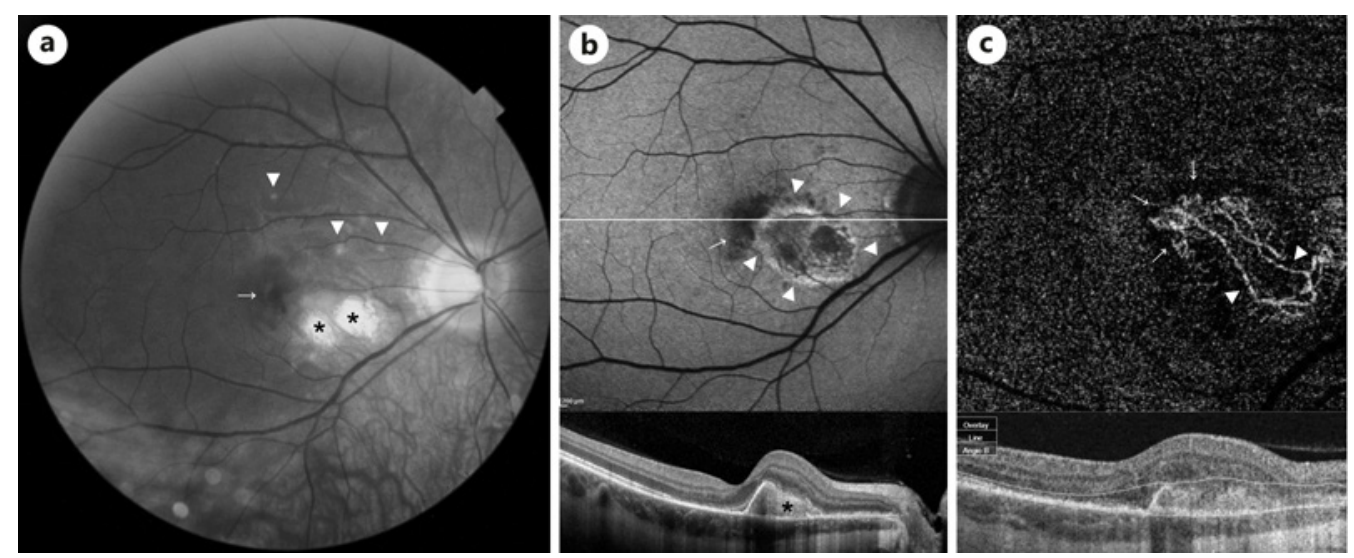

Fig. 2. a Follow-up 1 month later after 1 IVR and systemic corticosteroids infrared photography with resolution of subfoveal hemorrhage $(\rightarrow)$, demarcated dots $(\Delta)$, and spots $(*)$ in the posterior pole. $\mathbf{b}$ FAF with an almost normal hypo-FAF of the foveal depression $(\rightarrow$ ) next to a circumscribed hyper-FAF plaque consistent with the SD-OCT demonstrating prominent subretinal hyperreflective material (*) and neither SRF nor intravitreal inflammation. c Glomerular loops $(\rightarrow)$ with filamentous feeder vessels $(\Delta)$ in the SS-OCTA of an outer retina segmentation encompassing the whole lesion. IVR, intravitreal ranibizumab; SRF, subretinal fluid; FAF, fundus autofluorescence; SD-OCT, spectral domain-optical coherence tomography; SS-OCTA, swept source-optical coherence tomography angiography. 

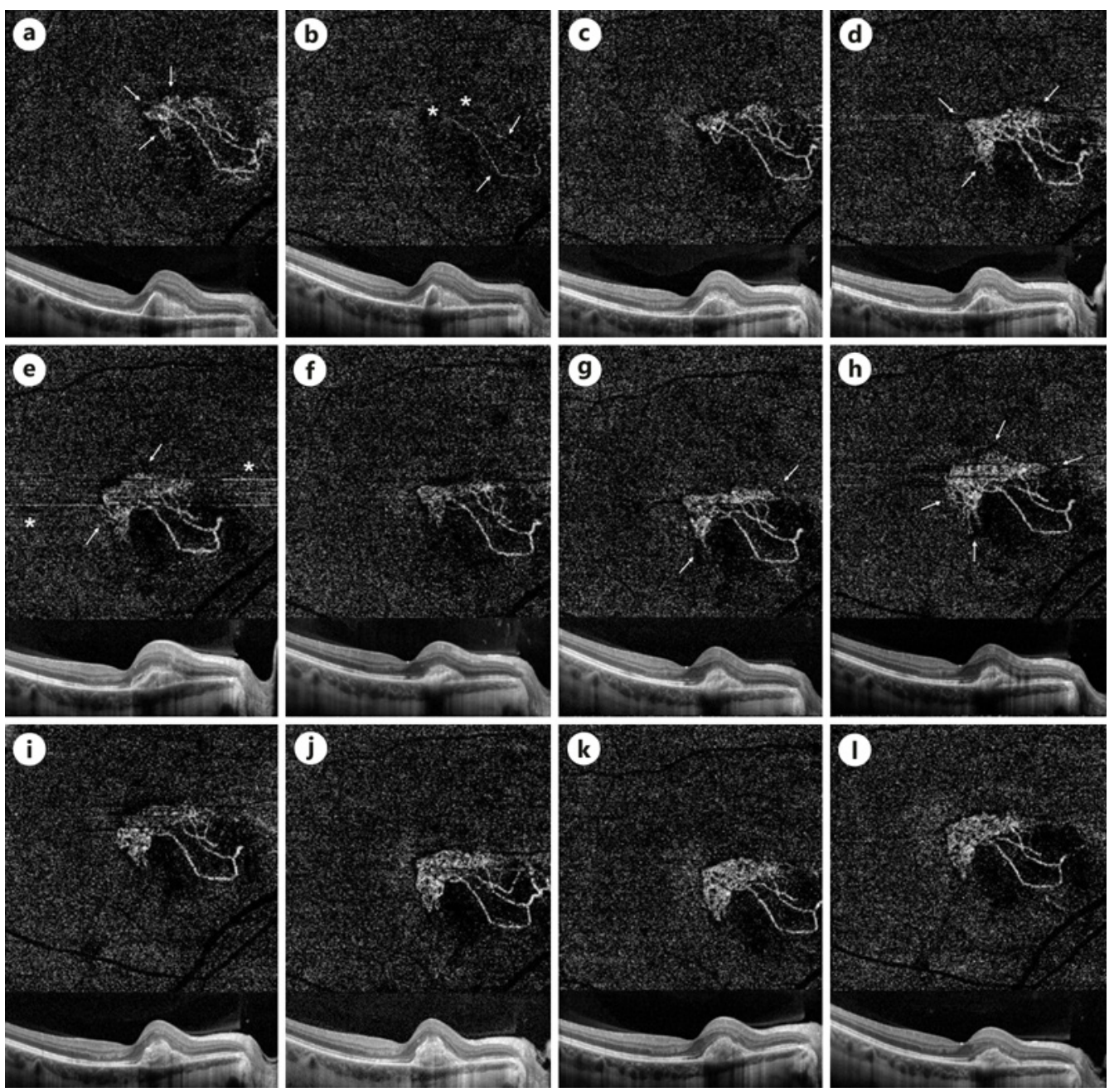

Fig. 3. a Follow-up comparing functional SS-OCTA en face scans with structural SD-OCT B-scans over time. b Second IVR based on small vessels at the tip of the CNV $(\rightarrow) 1$ month after initial presentation. c Low flow in the feeder trunks $(\rightarrow)$ and no flow $(*)$ in the neovascular network 1 week later. $\mathbf{d}$ Recirculation of the pre-existing CNV with regression of vessel termini as compared to image A after another month. e Third IVR based on new vessel formation and increased vessel density $(\rightarrow)$. $\mathbf{f}$ Fourth IVR due to ongoing neovascular growth $(\rightarrow)$ besides white band artefacts $\left({ }^{*}\right)$. $\mathbf{g}$ Regression of the network 1 month later. $\mathbf{h}$ Fifth IVR due to new capillaries at the lesion border similar to image $\mathrm{D}$ and as opposed to image F $(\rightarrow)$. Numerous new branches $(\rightarrow)$ led to the sixth IVR No sign of ongoing new vessel growth 1 month (i), 6 months (j), and 11 months ( $(\mathbf{k})$ after the last IVR. I No well-defined neovascular growth 33 months after initial presentation. SS-OCTA, swept source-optical coherence tomography angiography; SD-OCT, spectral domain-optical coherence tomography; IVR, intravitreal ranibizumab; CNV, choroidal neovascularization.

\section{Discussion}

Herein, we meticulously investigated CNV complexity in a rare chorioretinopathy pictured by SS-OCTA over a long observation period. SS-OCTA initially isolated the CNV membrane from other pathologies and was superior to structural SD-OCT in monitoring treatment. In contrast to another similar case report published in 2016, we were able to demonstrate the benefits of SS-OCTA not only for the detection of CNV secondary to PIC but particularly for follow-up and the management of therapeutic injections [9]. 
Inflammatory CNV are typically expressed as type 2 lesions with early leakage and welldefined borders in FA. Conventional angiography - although considered as gold standard for the diagnosis of CNV - is not recommended as the primary imaging tool at follow-up due to its invasive and time-consuming nature. SRF accumulation in OCT B-scans is generally but not necessarily always a reliable predictor for CNV activity especially in inflammatory neovascularization.

SS-OCTA was immensely helpful to determine the presence and alteration of CNV morphology. It enables the detection of blood flow and the visualization of vascular complexity. Previous descriptions of different CNV structures in OCTA and their incorporation into clinical practice have been investigated with varying results [10]. Coscas et al. [11] came up with a qualitative OCTA classification algorithm based on the presence or absence of 5 characteristics (branching capillaries, peripheral loops or arcades, anastomoses, filamentous vessels, nonvascularized halos) for activity of CNV in age-related macular degeneration. Al-Sheikh and her group compared biomarkers of active lesions before and after treatment to quiescent lesions and quantified pattern complexity by the fractal dimension [12]. Ichiyama et al. [13] investigated vascular density in CNV secondary to age-related macular degeneration and found good agreement for duration of remission and hence activity. The common sense of all research looking into a relation between the CNV morphology in OCTA and disease activity is to separate new formed vessels from more established mature vessels. Spaide elucidated the present possibility of anti-vascular endothelial growth factor (anti-VEGF) treatment to redirect the CNV process by controlling its growth rather than destroying it [14]. In this case, SS-OCTA en face images highlighted the transformation of the CNV architecture based on a qualitative assessment of flow pattern and density following antiangiogenic treatment. It will contribute to a deeper understanding of neovascular activity and might facilitate differentiation between active and regressed lesions without the necessity of repeated dye-dependent angiography [15].

In addition, we showed the successful treatment of a neovascularization due to PIC by combining a pro re nata regimen of intravitreal anti-VEGF and oral corticosteroids. Considering the variability of the disease, therapeutic management ranges from observation to extensive treatment options including systemic immunosuppression, intravitreal injections, photodynamic therapy, or submacular surgery [1]. Few papers have been published on the optimal approach toward PIC complicated by CNV. A prospective study with considerable high numbers of eyes affected primarily with PIC was recently published by Pohlmann et al. [5]. They separated their cohort into 2 groups: 1 treated with immunosuppressants, and another, control group. All eyes with secondary CNV received anti-VEGF independently of the underlying therapeutic regimen. A positive treatment effect on SRF and hence disease activity was concluded by the authors. In the light of the above, the bilateral mechanism of systemic anti-inflammatory and topic antiangiogenic drug administration seemed to be a promising approach and was effective in this case.

In conclusion, SS-OCTA was supportive to differentiate CNV from inflammatory lesions in the beginning and provided clinically relevant information regarding treatment strategies. As a noninvasive, repeatable, and fast imaging tool, it should be implemented in routine multimodal imaging procedures enabling clinicians to detect disease activity with more certainty. 


\section{Statement of Ethics}

All procedures performed in studies involving human participants were in accordance with the ethical standards of the institutional and/or national research committee and with the 1964 Helsinki Declaration and its later amendments or comparable ethical standards. For this type of study, formal consent is not required. The patient gave her written informed consent to publish the case.

\section{Conflict of Interest Statement}

The authors have no conflicts of interest to declare.

\section{Funding Sources}

This research was performed as part of the employment of the authors, namely, the Rudolf Foundation Hospital and the Karl Landsteiner Institute for Retinal Research and Imaging. It did not receive any specific grant from funding agencies in the public, commercial, or not-for-profit sectors.

\section{Author Contributions}

M.S. is the lead author and guarantor: conception, design, data acquisition, interpretation of data, and drafting of the article. J.F. and A.M.H.: data acquisition, analysis and interpretation of data, and drafting of the article. K.K.: interpretation of data and thorough revision of the article. S.A.S.: conception, drafting of the article, and critical revision. All authors read and approved the final version of the manuscript.

\section{References}

1 Ahnood D, Madhusudhan S, Tsaloumas MD, Waheed NK, Keane PA, Denniston AK. Punctate inner choroidopathy: a review. Surv Ophthalmol. 2017;62(2):113-26.

2 Gerstenblith AT, Thorne JE, Sobrin L, Do DV, Shah SM, Foster CS, et al. Punctate inner choroidopathy: a survey analysis of 77 persons. Ophthalmology. 2007;114(6):1201-4.

3 Watzke RC, Packer AJ, Folk JC, Benson WE, Burgess D, Ober RR. Punctate inner choroidopathy. Am J Ophthalmol. 1984;98(5):572-84.

4 Zhang X, Zuo C, Li M, Chen H, Huang S, Wen F. Spectral-domain optical coherence tomographic findings at each stage of punctate inner choroidopathy. Ophthalmology. 2013;120(12):2678-83.

5 Pohlmann D, Pleyer U, Joussen AM, Winterhalter S. Immunosuppressants and/or antivascular endothelial growth factor inhibitors in punctate inner choroidopathy? Follow-up results with optical coherence tomography angiography. Br J Ophthalmol. 2019;103(8):1152-7.

6 Ahmed D, Stattin M, Graf A, Forster J, Glittenberg C, Krebs I, et al. Detection of treatment-naive choroidal neovascularization in age-related macular degeneration by swept source optical coherence tomography angiography. Retina. 2017;38(11):2143-214.

7 Liang MC, de Carlo TE, Baumal CR, Reichel E, Waheed NK, Duker JS, et al. Correlation of spectral domain optical coherence tomography angiography and clinical activity in neovascular age-related macular degeneration. Retina. 2016;36(12):2265-73.

8 Pohlmann D, Pleyer U, Joussen AM, Winterhalter S. Optical coherence tomography angiography in comparison with other multimodal imaging techniques in punctate inner choroidopathy. Br J Ophthalmol. 2019;103(1): 60-6.

9 Nakao S, Kaizu Y, Oshima Y, Sakamoto T, Ishibashi T, Sonoda KH. Optical coherence tomography angiography for detecting choroidal neovascularization secondary to punctate inner choroidopathy. Ophthalmic Surg Lasers Imaging Retina. 2016;47(12):1157-61.

\section{Karger'}


10 Stattin M, Forster J, Daniel A, Graf A, Krepler K, Ansari-Shahrezaei S. Relationship between neovascular density in swept source-optical coherence tomography angiography and signs of activity in exudative age-related macular degeneration. J Ophthalmol. 2019;2019:4806061.

11 Coscas GJ, Lupidi M, Coscas F, Cagini C, Souied EH. Optical coherence tomography angiography versus traditional multimodal imaging in assessing the activity of exudative age-related macular degeneration: a new diagnostic challenge. Retina. 2015;35(11):2219-28.

12 Al-Sheikh M, Iafe NA, Phasukkijwatana N, Sadda SR, Sarraf D. Biomarkers of neovascular activity in age-related macular degeneration using optical coherence tomography angiography. Retina. 2018;38(2):220-30.

13 Ichiyama Y, Sawada T, Ito Y, Kakinoki M, Ohji M. Optical coherence tomography angiography reveals blood flow in choroidal neovascular membrane in remission phase of neovascular age-related macular degeneration. Retina. 2017;37(4):724-30.

14 Spaide RF. Choroidal neovascularization. Retina. 2017;37(4):609-10.

15 Levison AL, Baynes KM, Lowder CY, Kaiser PK, Srivastava SK. Choroidal neovascularisation on optical coherence tomography angiography in punctate inner choroidopathy and multifocal choroiditis. Br J Ophthalmol. 2017;101(5):616-22. 